\title{
Preliminary Study on Aerodynamic Control of High-Angle-of-Attack Slender Body Using Blowing from Penetrating Flow Channels
}

\author{
Ayane Sato, ${ }^{1}$ Hiroyuki Nishida, ${ }^{2}$ and Satoshi Nonaka ${ }^{3}$ \\ ${ }^{1}$ Department of Mechanical Systems Engineering, Tokyo University of Agriculture and Technology, 2-24-16 Naka-cho, \\ Koganei, Tokyo 184-8588, Japan \\ ${ }^{2}$ Institute of Engineering, Tokyo University of Agriculture and Technology, 2-24-16 Naka-cho, Koganei, Tokyo 184-8588, Japan \\ ${ }^{3}$ Institute of Space and Astronautical Science, Japan Aerospace Exploration Agency, 3-1-1 Yoshinodai, Chuo-ku, \\ Sagamihara, Kanagawa 252-5210, Japan \\ Correspondence should be addressed to Hiroyuki Nishida; hnishida@cc.tuat.ac.jp
}

Received 8 August 2016; Accepted 24 October 2016

Academic Editor: Antonio Ficarella

Copyright (C) 2016 Ayane Sato et al. This is an open access article distributed under the Creative Commons Attribution License, which permits unrestricted use, distribution, and reproduction in any medium, provided the original work is properly cited.

The objective of this study is to experimentally verify a new aerodynamic control concept of a high-angle-of-attack slender body. In the concept, penetrating flow channels are installed to the apex of the slender body. The blowing or suction is generated at the channel exits in response to the surface pressure distribution. First, the effects of the flow channels on the aerodynamic characteristics are experimentally investigated in a low-speed wind tunnel. The result shows the Suction-Blowing type channel is the most effective because its control effect does not reduce even in higher mainstream flow velocity. The peak value of the side force and yawing moment can be reduced by up to $64 \%$ and $49 \%$, respectively. In addition, visualization of the surface flow pattern by the oil flow method shows that the Suction-Blowing type channel makes not only the primary separation line on the body side but also the secondary separation line on the body back become symmetric.

\section{Introduction}

When a slender body, which is a typical body shape of a forebody of air planes and rockets, flies at high angle of attack, the flow field around the slender body intricately changes depending on the body shape, the Reynolds number of main flow, and angle of attack. At that situation, asymmetric vortices are formed behind the slender body (Figure 1) and generate the side force whose amplitude is up to the half of the normal force acting on the body. It causes severe loss of the attitude stability of the body $[1,2]$. Aerodynamic control surfaces such as a vertical tail fin cannot work effectively at high angle of attack because they are in the wake flow; therefore, it is one of promising solutions for this problem to utilize flow control devices aiming to control the vortex structure.

Various devices have been developed to control the flow field around a slender body. For example, strakes at the body apex are one of well-known passive flow control devices $[3,4]$.
It was shown that the strakes can make the flow separation point symmetric and suppress the side force and yawing moment. Their advantages are simplicity of structure and easy installation; however, they have also disadvantages such as increase of drag and structural weight. Micro blowing is one of well-known active flow control devices [5-7]. It is installed to change the vortex structure around the body by blowing gas from small holes at the body apex. It can actively control the direction of the side force and yawing moment by blowing gas from the starboard or port side blowing hole. The side force obtained by the micro blowing is larger than the momentum of the jet, therefore the control efficiency is high. However, it has not been put into practical use yet due to its complex mechanical structure, that is, needs for high pressure gas tanks and complex valve system. Recently, a plasma actuator gets much attention as one of promising active flow control devices $[8,9]$. It is composed of two electrodes separated by a dielectric. When an AC high voltage with several 


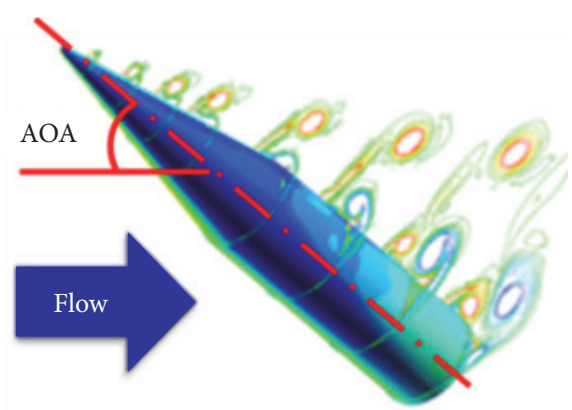

FIGURE 1: Asymmetric separation vortices.

$\mathrm{kV}$ and $\mathrm{kHz}$ is applied, the surface flow along the dielectric surface is generated. Its advantages are no mechanical moving parts, active controllability by electrical signal, and thin structure. Because of these advantages, it can overcome the difficulties of conventional flow control devices. In previous studies using burst-mode actuation of plasma actuators, one pair of plasma actuator was installed at the body apex, and the side force at high angle of attack was continuously changed by changing the bust ratio between the starboard and port side actuator $[10,11]$. However, the induced flow is weak (typically up to only several $\mathrm{m} / \mathrm{s}$ ), and the control effect severely reduces in higher main flow velocity $[12,13]$.

In the past, a two-dimensional slit (penetrating flow channel) was proposed and studied to control the boundary layer and wake flow structure around a circular cylinder $[14,15]$. The width of the flow channel was $8 \%$ or $18.5 \%$ of the cylinder diameter, and flow control experiments were conducted changing the tilt angle of the flow channel against the main flow direction. The results successfully showed the effectiveness of the penetrating flow channel; when the tilt angle of the channel is in a range from 0 to 40 degs., the drag coefficient decreases from 20 to $30 \%$ due to not only reduction of the projected area against the flow but also recovery of the back pressure. When the tilt angle is in a range from 80 to 90 degs., boundary layer suction is made at one side of the flow channel and at the other end, blowing is generated; as a result, the shedding of vortices can be controlled.

Based on the above-mentioned background, we propose to install penetrating flow channels to the apex of a slender body for suppression of the side force. The channel flow is generated by the pressure distribution on the body surface, and it generates a blowing and suction on the body surface. It is easy to be installed because of no need of complicated mechanical parts such as high pressure gas tanks. The behavior of its blowing changes in response to the change in the pressure distributions on the surface. Therefore, the penetrating flow channel is passive device, but it is expected to control the flow field automatically and flexibly even when the flow condition changes. Furthermore, it is expected that the control effect does not reduce in higher speed main flow because higher flow speed generates larger pressure difference between two ends of the flow channel.

In this study, a series of preliminary wind tunnel experiment is conducted for a high-angle-of-attack slender body with penetrating flow channels. The objective is to experimentally investigate the aerodynamic control effects of the flow channel for suppressing the high-angle-of-attack side force and yawing moment. Following experiments are conducted in this study:

(1) Aerodynamic characteristics measurements of the slender body model with penetrating flow channels in a low-speed wind tunnel.

(2) Visualization of surface flow pattern on the body by the oil flow method.

\section{Flow Control Concepts Using Penetrating Flow Channel}

We propose two types of flow control concept using penetrating flow channel at the body apex. One is to generate the channel flow utilizing the pressure difference between the stagnation point with higher pressure and the body side or back with lower pressure; Figure 2 shows the schematic figure of this flow control concept (cross-sectional view). In Figure 2 , the vortex structure without flow channels are indicated by doted lines; the vortex structure is asymmetric due to the high angle of attack. Suction appears at the channel exit in the stagnation point, and on the other hand, blowing appears at the two channel exits on the body side (or back). The blowing becomes stronger on the side where the vortex is closer to the body because of later flow separation. Therefore, it is expected that the vortex closer to the body is pushed away or the flow separation point moves forward by the stronger blowing (depicted by the red line in Figure 2), and as a result, the vortex structure becomes more symmetric; that is, the side force and yawing moment can be suppressed. We call this concept the B-B type because blowing appears at both channel exits.

The other is to generate channel flow utilizing the pressure difference between the starboard and port side of the body as shown in Figure 3; when the separation flow is asymmetric, the flow separation point is also different between on the starboard and port side of the body, and pressure difference is generated. It is expected that the suction appears on the body side with earlier flow separation, and the blowing appears on the other body side. As a result, the separation points can be adjusted into symmetric positions. We call this concept the $\mathrm{S}-\mathrm{B}$ type because both suction and blowing appear.

In our concepts, the flow by the channel automatically changes in response to the flow structure around the body, and therefore, it is expected that our flow concept can work effectively in different flow condition without any active control.

\section{Experimental Methods and Conditions}

The experiments are conducted using the planet environmental wind tunnel in ISAS (Institute of Space and Astronautical Science)/JAXA (Japan Aerospace Exploration Agency). This is the Goettingen type low-speed wind tunnel and the flow velocity can be controlled in the range from 10 to $50 \mathrm{~m} / \mathrm{s}$. The test section is open type and its diameter is $1.6 \mathrm{~m}$. The testing model configuration is shown in Figure 4. It is a simple cone-cylinder configuration and its slenderness ratio 


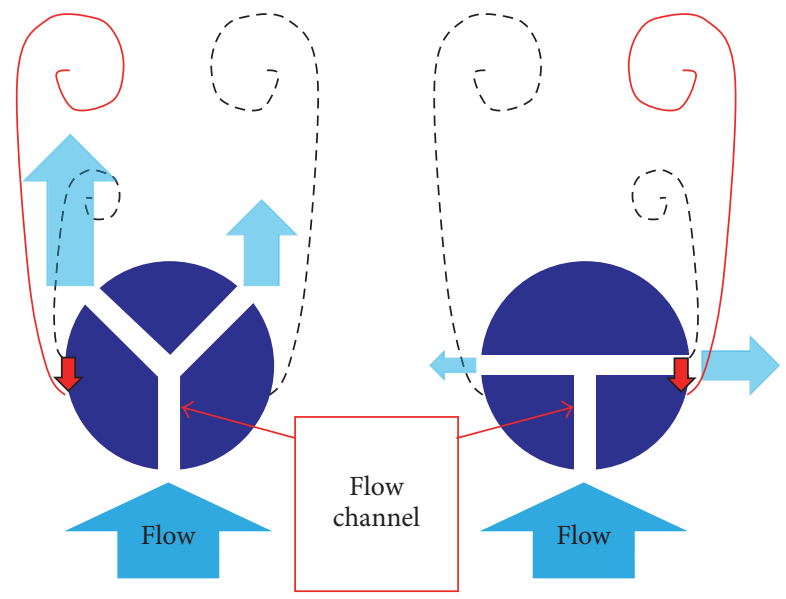

Figure 2: B-B type flow channel (cross-sectional view of the body apex).

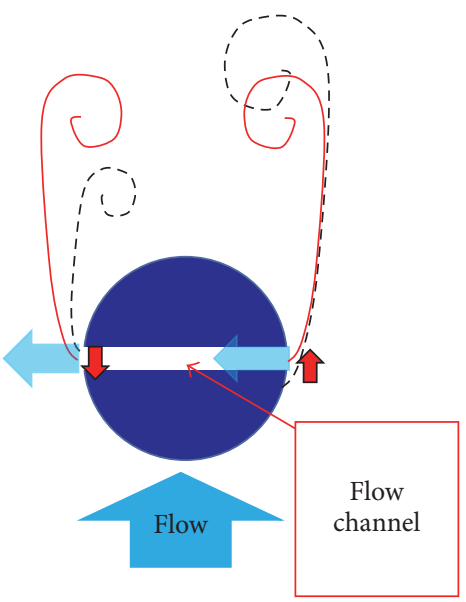

FIGURE 3: S-B type flow channel (cross-sectional view of the body apex).

is 11.5. Figure 5 shows the 3D-CAD figure of the model and its enlarged view of the body apex where flow channels are installed. The testing model is made by the stereolithographic technique.

Table 1 shows experimental cases of flow channel. Three types of flow channel shape are investigated: two types of the B-B type concept ( $\mathrm{T}$ - and Y-shaped channel) and the S-B type channel. In addition, five different channel positions are investigated; $x / L=0.035,0.070,0.10,0.14$, and 0.17 from the body tip, where $L$ is the model total length. The experimental case can be changed by closing unnecessary channels using clay.

The aerodynamic force acting on the model is measured using a six-component internal balance. The measurement is conducted changing the angle of attack from 0 to 90 degs., and the data is obtained three times for each case of angle of attack to estimate the measurement error. The aerodynamic forces are nondimensionalized using the mainstream dynamic pressure and the cross-sectional area of the model base. In this study, the normal force coefficient $C_{N}$, the

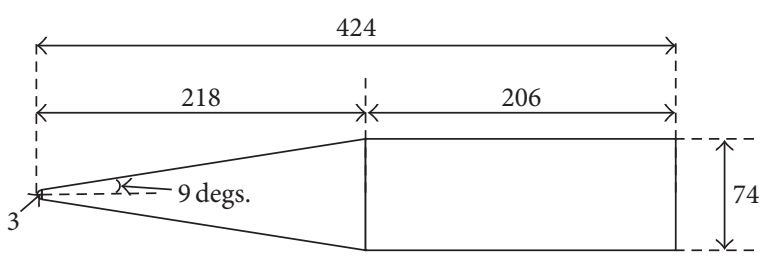

FIGURE 4: Wind tunnel testing model configuration.

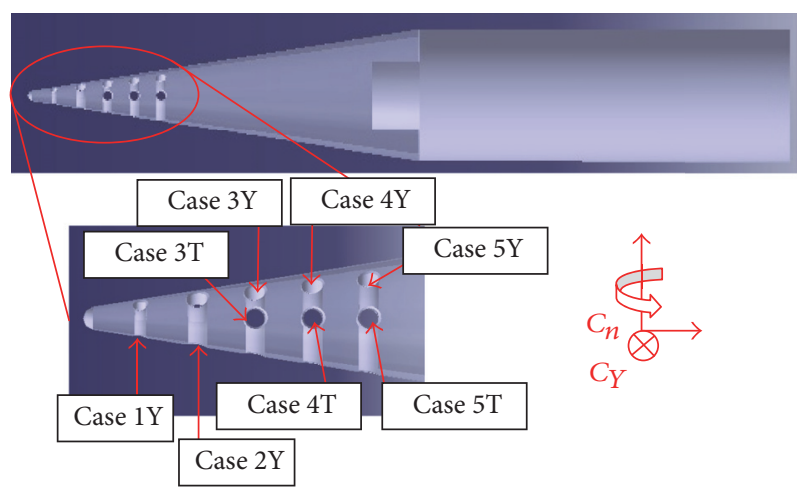

Figure 5: Penetrating flow channels.

side force coefficient $C_{Y}$ and yawing moment coefficient $C_{n}$ are focused to investigate the effects of the flow channel. The Reynolds number is $7.8 \times 10^{4}, 1.2 \times 10^{5}$, and $1.7 \times 10^{5}$ (mainstream velocity is 16,24 , and $35 \mathrm{~m} / \mathrm{s}$, resp.). In addition to the aerodynamic force measurement, the surface flow pattern is visualized by the oil flow method. A mixture of oil and some pigments is painted on the model surface, and the surface flow can be estimated from the oil film pattern. The oil is liquid paraffin, and the pigment is titanium oxide. In the experiment for the oil flow visualization, the Reynolds number is $1.7 \times 10^{5}$ (main flow speed is $35 \mathrm{~m} / \mathrm{s}$ ).

\section{Experimental Results and Discussions}

4.1. Aerodynamic Force Characteristics. First, the effects of the B-B type flow channels on the aerodynamic characteristics are discussed. The normal force coefficients are shown in Figures 6 and 7, and the side force coefficients are shown in Figures 8 and 9. The flow velocity is $16 \mathrm{~m} / \mathrm{s}$ in Figures 6 and 8 and $24 \mathrm{~m} / \mathrm{s}$ in Figures 7 and 9 . In addition, the yawing moment coefficients are shown in Figures 10 and 11, in which the moment center is at $65 \%$ length from the model tip. As apparently observed in these figures, the effects of the channels on the normal force are much smaller; this indicates that the channels do not change the size of the wake region but modify the asymmetry of the vortex structure. From this point forward in this section, we focus on the side force and yawing moment.

For the case without the flow channel (black lines in the figures), when the angle of attack becomes larger, the side force starts to appear at the angle of attack larger than about 40 degs. which is about twice the body apex angle and then reaches the peak value at 50 degs. (up to the half of the 
TABLE 1: Flow channel configurations.

\begin{tabular}{|c|c|c|c|c|c|c|c|c|c|c|c|}
\hline$x / L$ & 0.035 & 0.070 & \multirow{2}{*}{\multicolumn{3}{|c|}{$5 \mathrm{~mm}$}} & \multicolumn{3}{|c|}{0.14} & \multicolumn{3}{|c|}{0.17} \\
\hline Channel diameter $d$ & $3 \mathrm{~mm}$ & $5 \mathrm{~mm}$ & & & & \multicolumn{3}{|c|}{$5 \mathrm{~mm}$} & \multicolumn{3}{|c|}{$5 \mathrm{~mm}$} \\
\hline Case & $1 \mathrm{Y}$ & $2 \mathrm{Y}$ & $3 \mathrm{~T}$ & $3 Y$ & $3 S-B$ & $4 \mathrm{~T}$ & $4 \mathrm{Y}$ & $4 S-B$ & $5 \mathrm{~T}$ & $5 \mathrm{Y}$ & $5 S-B$ \\
\hline Channel configuration & $\boldsymbol{Y}$ & & 8 & $=$ & $\Omega$ & 7 & & 0 & 8 & 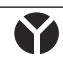 & 0 \\
\hline
\end{tabular}

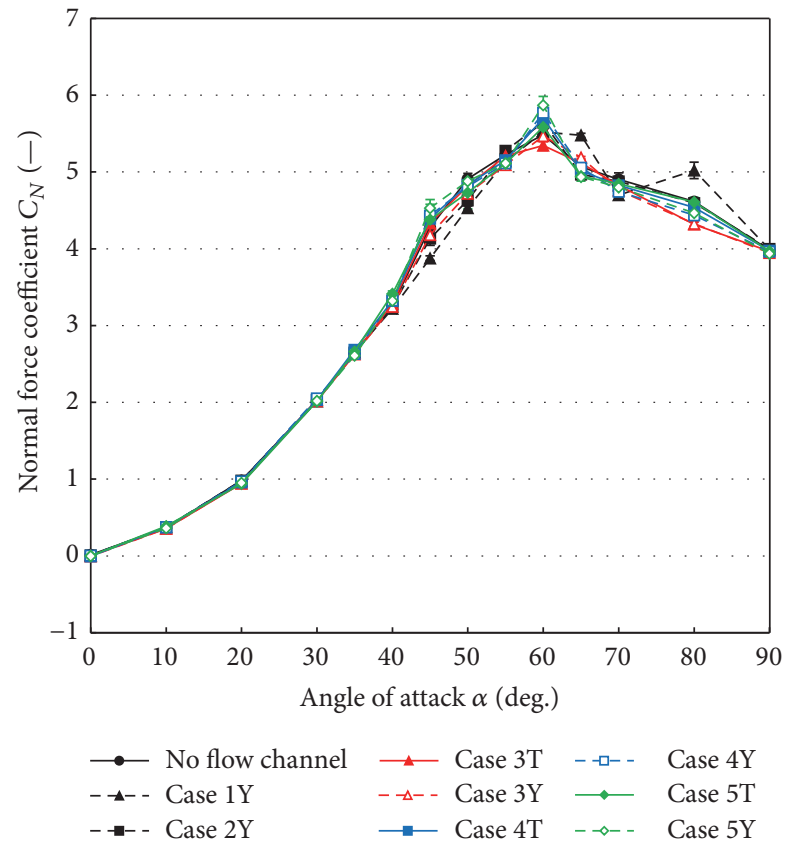

Figure 6: Normal force coefficient: B-B type channels, $16 \mathrm{~m} / \mathrm{s}$.

peak value of the normal force). After that, the side force drops to 0 at around 55 degs. and its sign is reversed at larger angle. Finally, the side force turns into zero at angle larger than 70 degs. because the flow behind the body becomes the vortex wake. This trend of the side force is qualitatively and quantitatively in good agreement with that reported by other research groups $[1,2,16]$. The behavior of the yawing moment is more complicated. It starts to appear at the angle in which the side force appears and changes its sign two times (of course, the behavior of the yawing moment depends on the moment center). As shown in Figures 8 and 9, the sign of the side force is reversed between the mainstream flow velocities of $16 \mathrm{~m} / \mathrm{s}$ and $24 \mathrm{~m} / \mathrm{s}$. This is because the vortex structure is inverted to right and left depending on the change in the mainstream condition.

Figures 12, 13, 14, and 15 show selected cases in which the side force or yawing moment is effectively suppressed. When the mainstream flow velocity is $16 \mathrm{~m} / \mathrm{s}$, in Figure 12 , it is shown that the case with the $1 Y$ channel, which is closest to the model tip, provides the best control effect to suppress the peak value of the side force; $59 \%$ of the peak value of the side force is reduced. When the mainstream flow velocity is $24 \mathrm{~m} / \mathrm{s}$, in Figure 13, the side force suppression effect of the $1 Y$ channel is apparently lost, and on the contrary, the $1 Y$ channel increases the side force. Instead of the $1 Y$ channel, the $3 \mathrm{~T}$ channel which is located at $10 \%$ length from the model

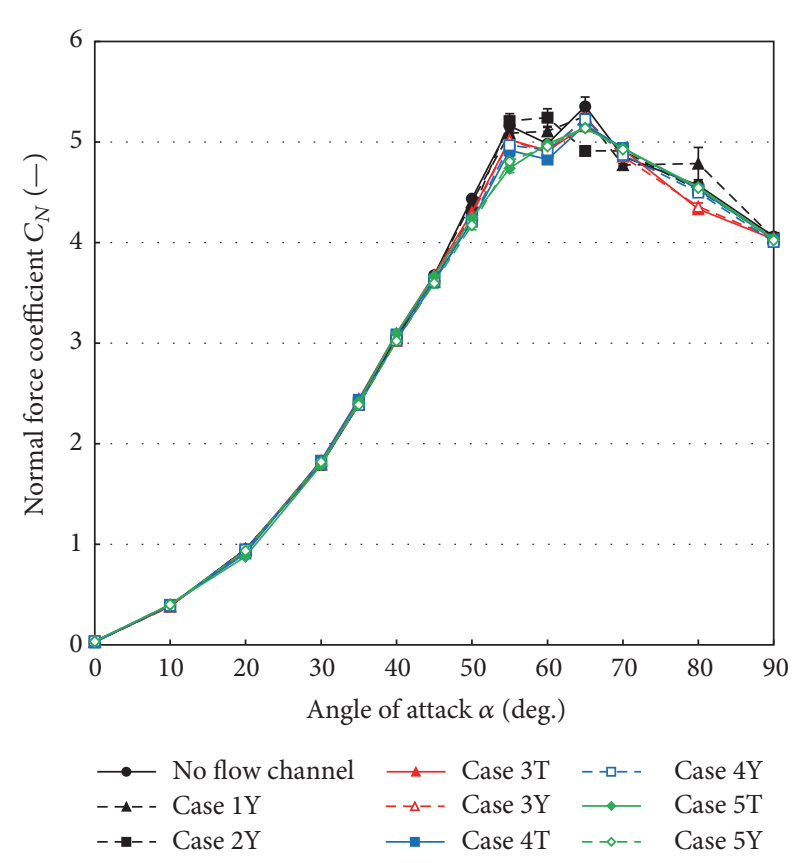

Figure 7: Normal force coefficient: B-B type channels, 24 m/s.

tip provides better control effect; the side force is reduced by about $19 \%$.

When the mainstream flow velocity is $16 \mathrm{~m} / \mathrm{s}$, it is shown in Figure 14 that the 1Y channel successfully suppresses the yawing moment as with the case of the side force. The yawing moment is suppressed by about $49 \%$, and the suppression effect can be obtained in wide range of the angle of attack. When the mainstream flow velocity increases to $24 \mathrm{~m} / \mathrm{s}$, the same tendency as in the side force (Figure 13) can be observed in the yawing moment (Figure 15). The yawing moment suppression by the $1 Y$ channel disappears, and on the other hand, the $3 \mathrm{~T}$ channel becomes the most effective for the yawing moment suppression; the peak value of the yawing moment can be suppressed by about $29 \%$.

Next, the results of the S-B type flow channels are shown. Figures 16 and 17 show the effects of the channels on the normal force. The side force is shown in Figures 18 and 19, and the yawing moment is shown in Figures 20 and 21. The mainstream flow velocity is $16 \mathrm{~m} / \mathrm{s}$ in Figures 16, 18, and 20 and $35 \mathrm{~m} / \mathrm{s}$ in Figures 17, 19, and 21. As in the case of the B-B type channel, the S-B type channels have little effect on the normal force.

When the mainstream flow velocity is $16 \mathrm{~m} / \mathrm{s}$, it can be observed in Figure 18 that the case of 5S-B type channel is the most effective to suppress the peak value of the side force, and the reduction of $45 \%$ is attained. When the mainstream 


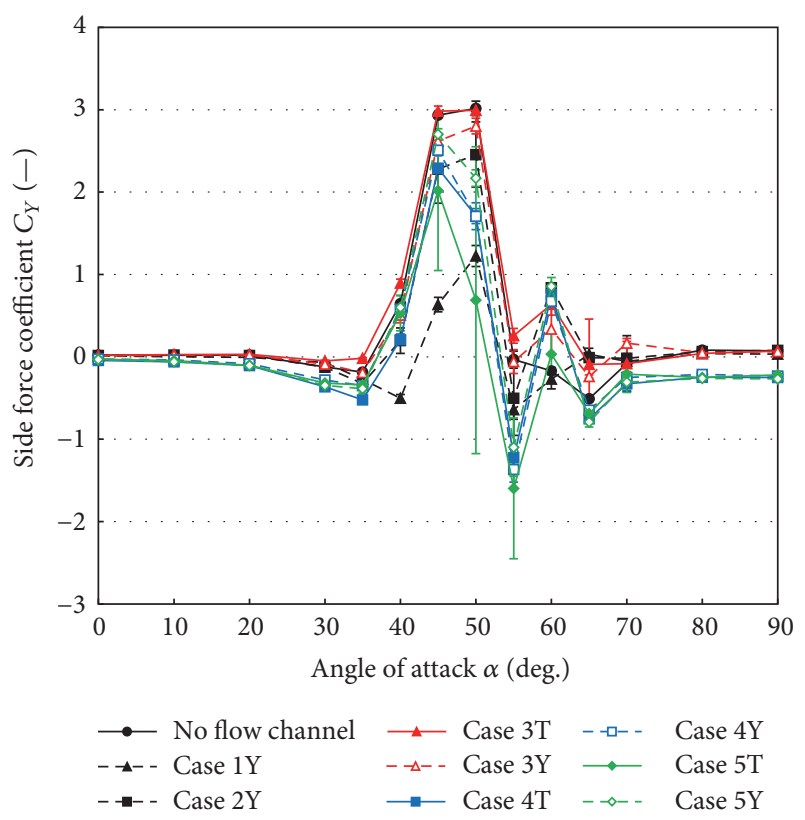

FIGURE 8: Side force coefficient: B-B type channels, $16 \mathrm{~m} / \mathrm{s}$.

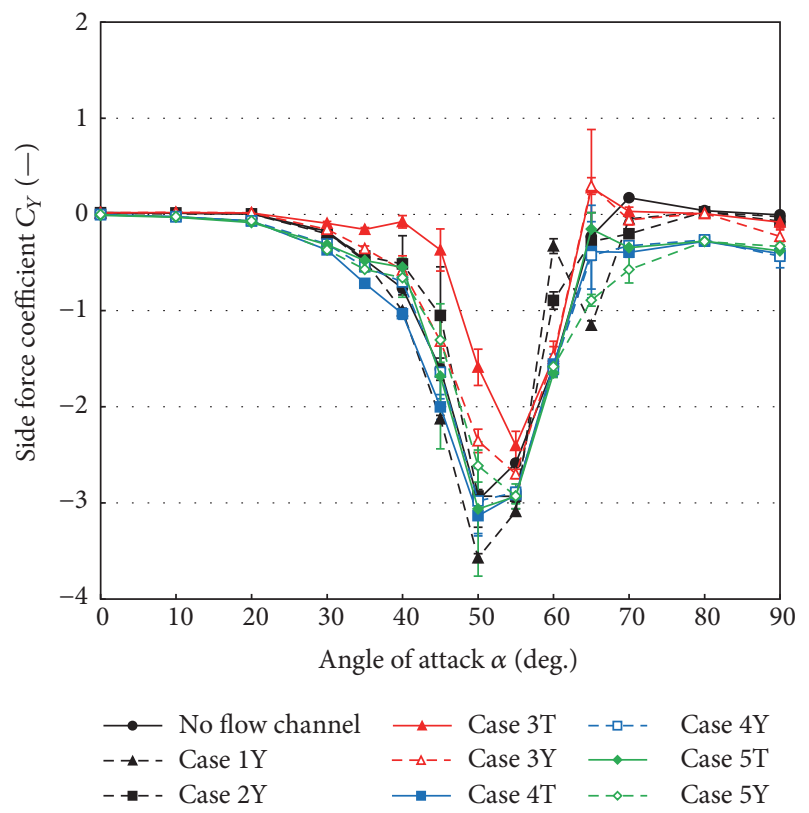

FIgURE 9: Side force coefficient: B-B type channels, $24 \mathrm{~m} / \mathrm{s}$.

flow velocity increases to $35 \mathrm{~m} / \mathrm{s}$ (as shown in Figure 19), the suppression rate of the side force peak value by the 5S-B type channel increases up to about $64 \%$; this is the highest suppression rate in all experimental cases. As shown in Figures 20 and 21, the yawing moment is also suppressed by the $5 \mathrm{~S}-\mathrm{B}$ type channel, and its suppression rate of the peak value is up to about $48 \%$ in the mainstream of $35 \mathrm{~m} /$ s.

As indicated in the third paragraph of this section, the flow control effects by the B-B type flow channel strongly depend on the mainstream flow velocity. This is because the

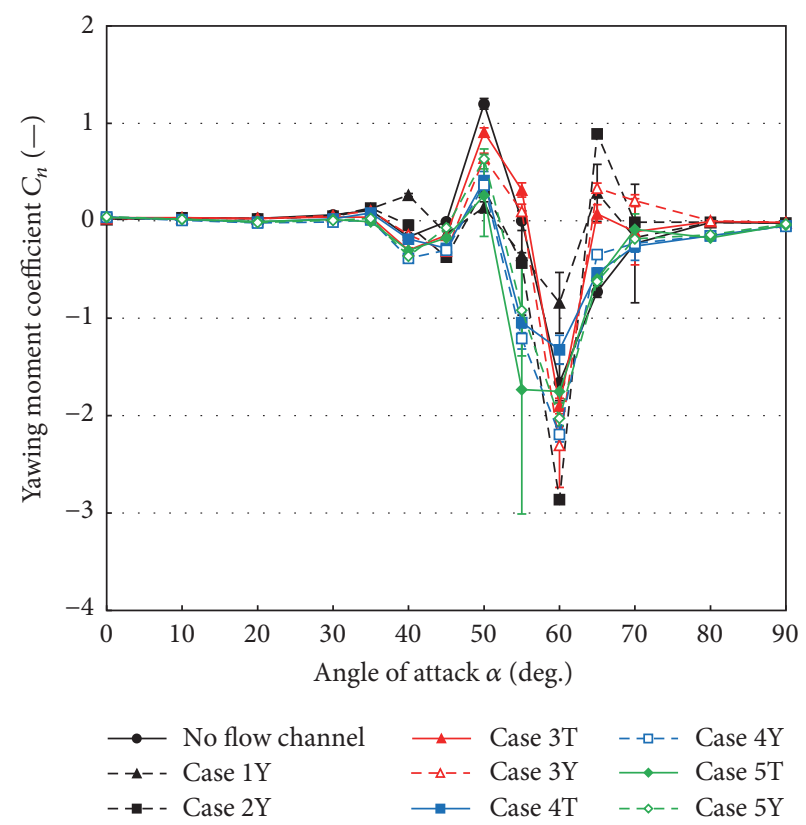

Figure 10: Yawing moment coefficient: B-B type channels, 16 m/s.

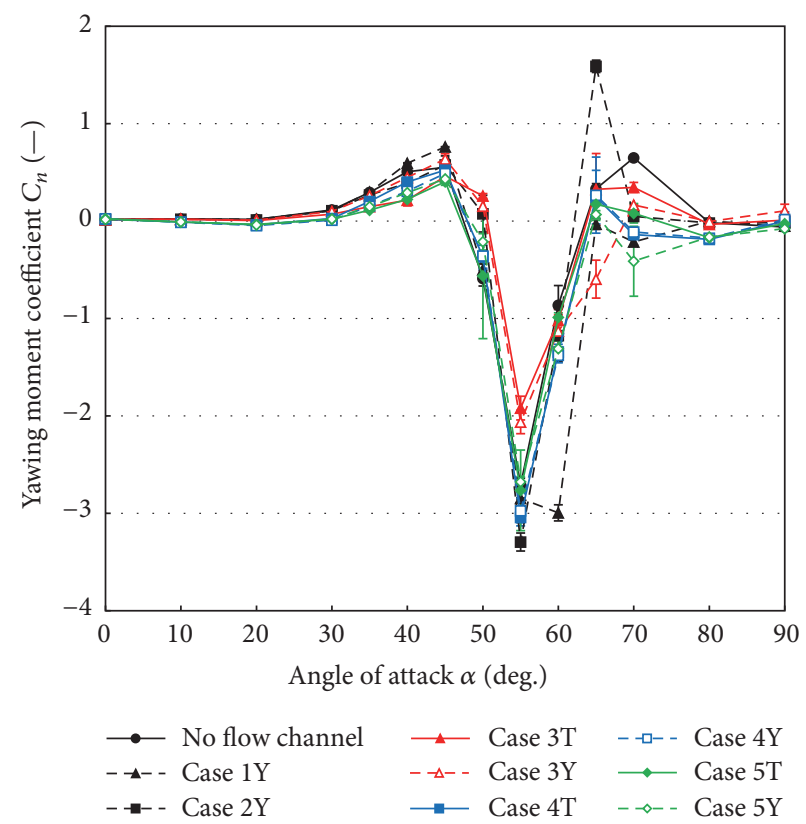

Figure 11: Yawing moment coefficient: B-B type channels, 24 m/s.

Reynolds number of the experiment is inside the transition range from laminar to turbulent (the typical critical Reynolds number is about $3.5 \times 10^{5}$ ), and even small changes in the flow velocity significantly modify the flow structure. For the B-B type channel, the positions and channel shapes should be carefully selected in response to the flow structure (more detailed discussions on the influence of the Reynolds number need further studies including precise flow visualization such as the Particle-Image-Velocimetry (PIV) measurement). In addition, the disadvantage of the $\mathrm{B}-\mathrm{B}$ type channel is that its effect reduces in higher flow velocity of the mainstream. On 


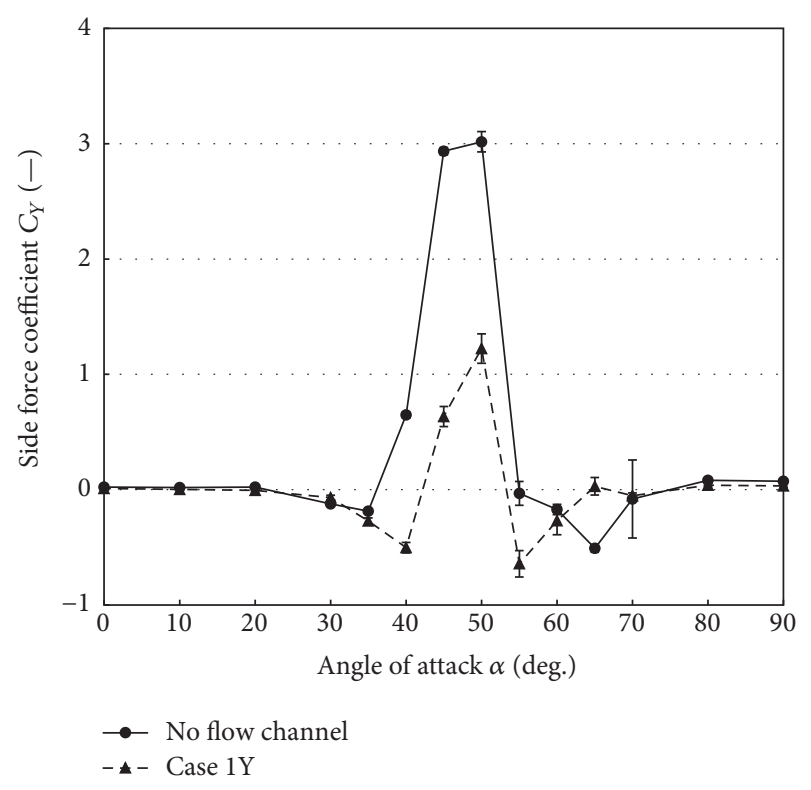

FIGURE 12: Side force coefficient: effective B-B type channels, $16 \mathrm{~m} / \mathrm{s}$.

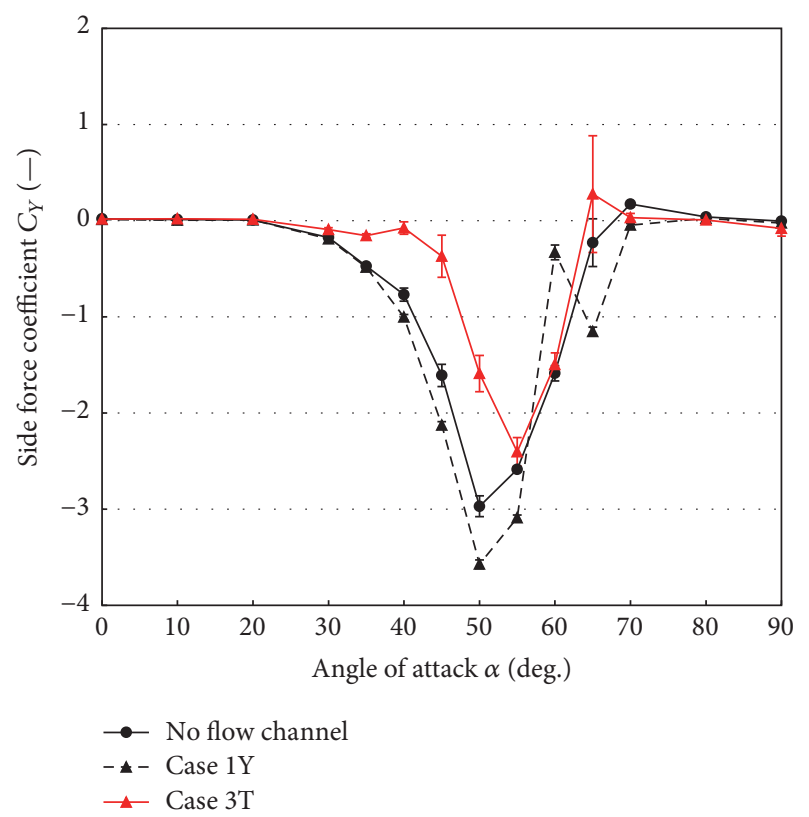

FigurE 13: Side force coefficient: effective B-B type channels, $24 \mathrm{~m} / \mathrm{s}$.

the other hand, for the case of the S-B type flow channel, even though the position of the channel should be appropriately selected, the suppression effect of the side force and yawing moment is better than that of the B-B type channels and, in addition, does not reduce in higher mainstream flow velocity. In this experiments, the S-B type channel at $17 \%$ position from the model tip (the 5S-B type channel) can provide good result at the wide range of the angle of attack and in both the lower and higher mainstream flow velocity. Note that the channels adopted in this study cannot remove the side force completely, and therefore, there is still room for improvement in location and shape of the channel.

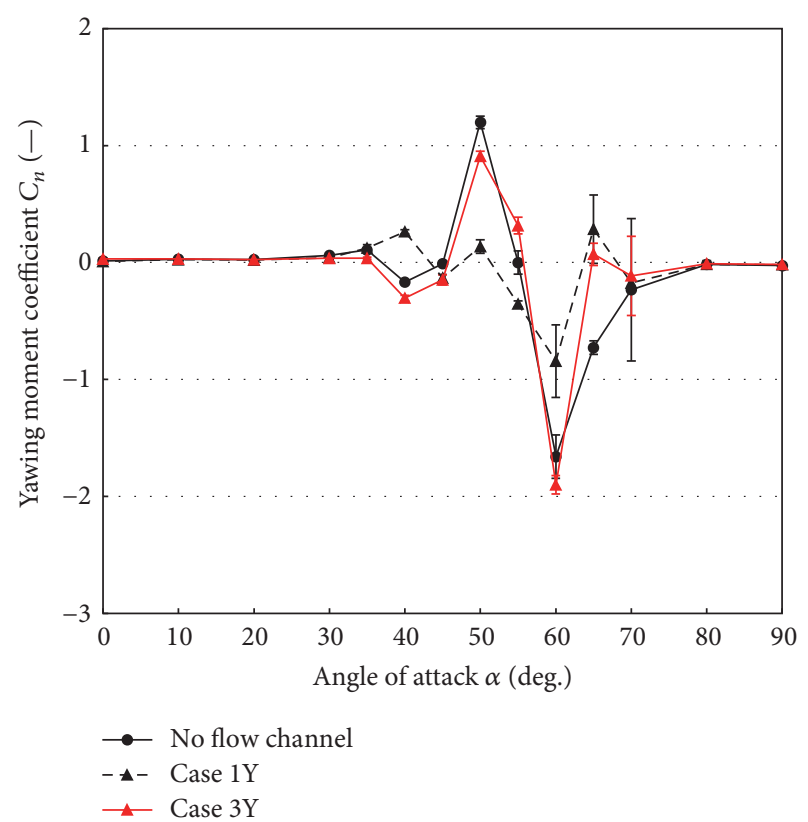

FIGURE 14: Yawing moment coefficient: effective B-B type channels, $16 \mathrm{~m} / \mathrm{s}$.

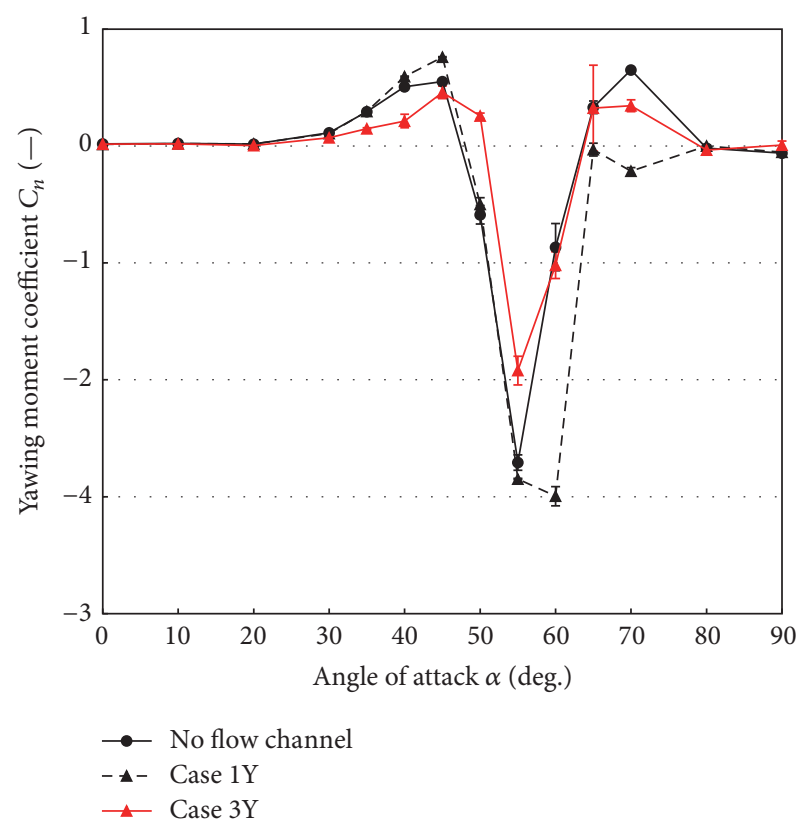

FIGURE 15: Yawing moment coefficient: effective B-B type channels, $24 \mathrm{~m} / \mathrm{s}$.

4.2. Surface Flow Visualization by Oil Flow Method. The flow pattern on the model surface is visualized for the case without the flow channel and with the 5S-B type flow channel which provides the best suppression result. The angle of attack is 50 degs. and the mainstream flow velocity is $35 \mathrm{~m} / \mathrm{s}$. Figures 22 and 23 show the starboard and port side view, and Figures 24 and 25 show the back side view; the case without the flow channel is in Figures 22 and 24, and with the 5S-B channel is in Figures 23 and 25. In the figures, the primary separation 


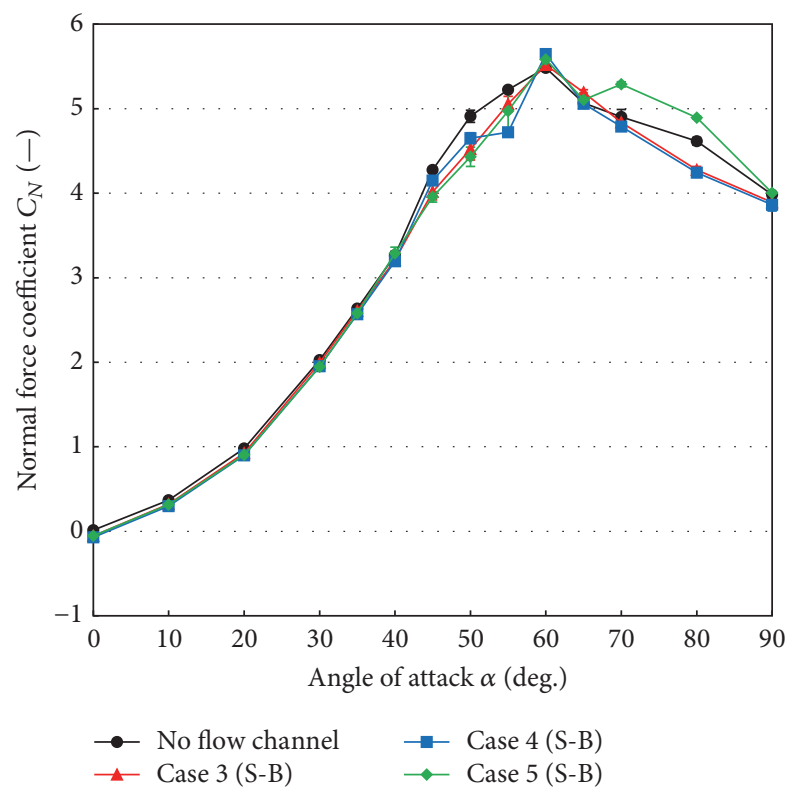

FIGURE 16: Normal force coefficient: S-B type channels, $16 \mathrm{~m} / \mathrm{s}$.

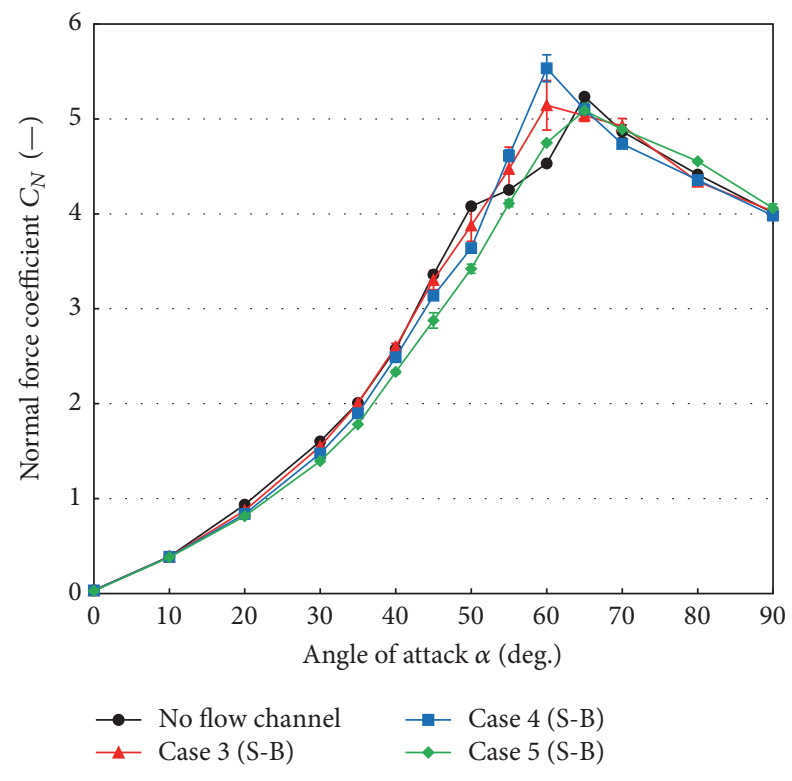

FIGURE 17: Normal force coefficient: S-B type channels, $35 \mathrm{~m} / \mathrm{s}$.

lines and secondary separation lines are indicated by red and blue lines, respectively (the reattachment line is near the downstream-side stagnation line $[17,18])$. In addition, the lines at circumferential angle of 90 degs. from the windward stagnation point are indicated by green dotted lines for the reference. Particularly, there is strong relationship between the primary separation line and vortex structure. The vortex at the side with later flow separation is closer to the model (see the schematic figure in Figures 2 and 3). The side force appears in the direction from the earlier separation side to the later separation side.

From the comparison between Figures 22 and 23, it can be observed that the flow channel makes the primary separation

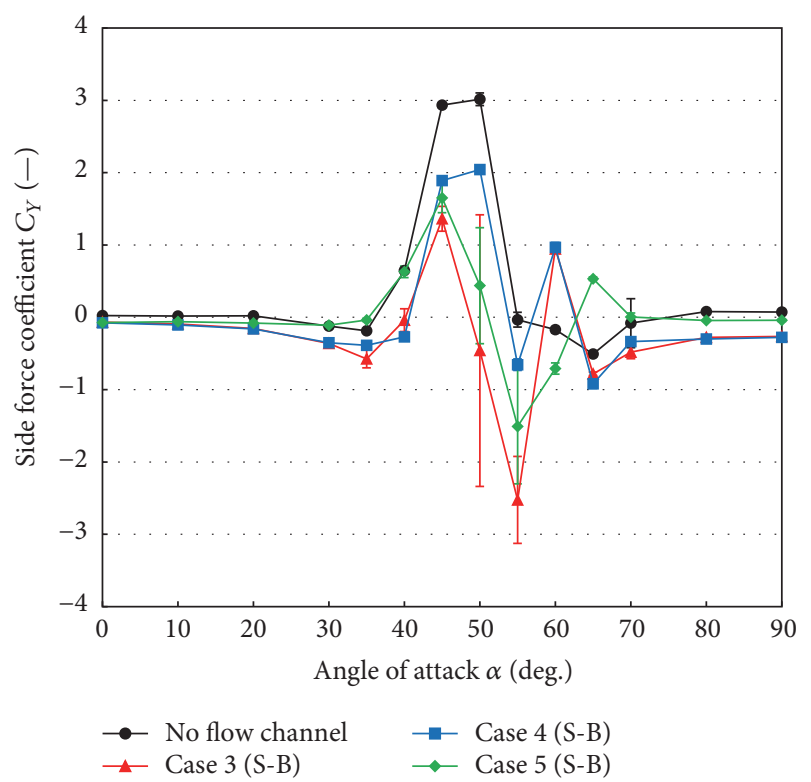

FIGURE 18: Side force coefficient: S-B type channels, $16 \mathrm{~m} / \mathrm{s}$.

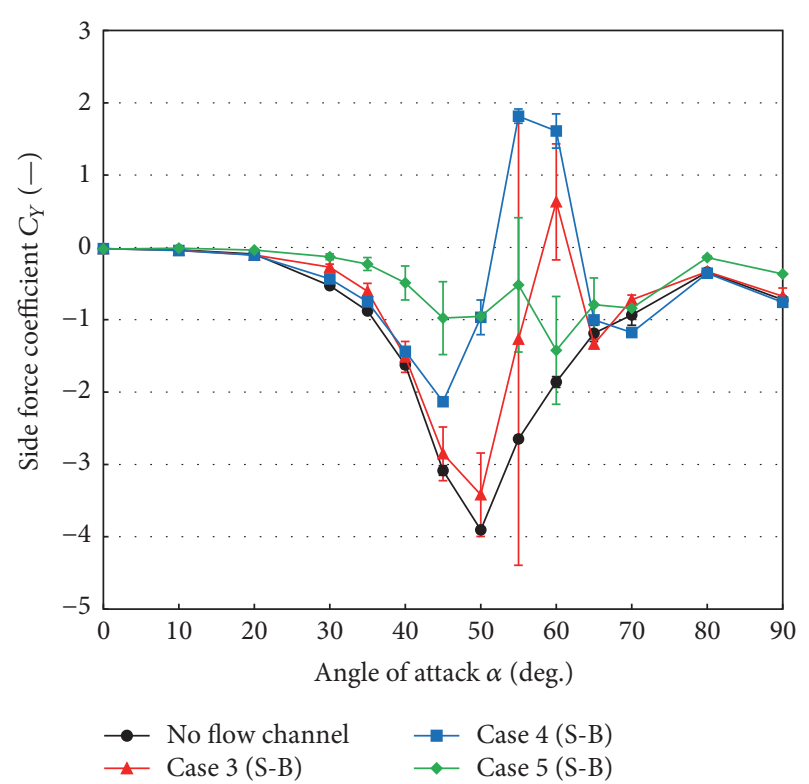

FIgURE 19: Side force coefficient: S-B type channels, $35 \mathrm{~m} / \mathrm{s}$.

earlier on both the starboard and port side of the model, and as a result, the starboard and port side position of the flow separation becomes more symmetric. Besides, from the comparison of back side flow patterns (Figures 24 and 25 ), it can be confirmed that the structure of the secondary separation lines also becomes much more symmetric by the installation of the flow channel.

As just described, the flow channel works to make not only the primary flow separation on the model side but also the secondary separation on the model back become symmetric. 


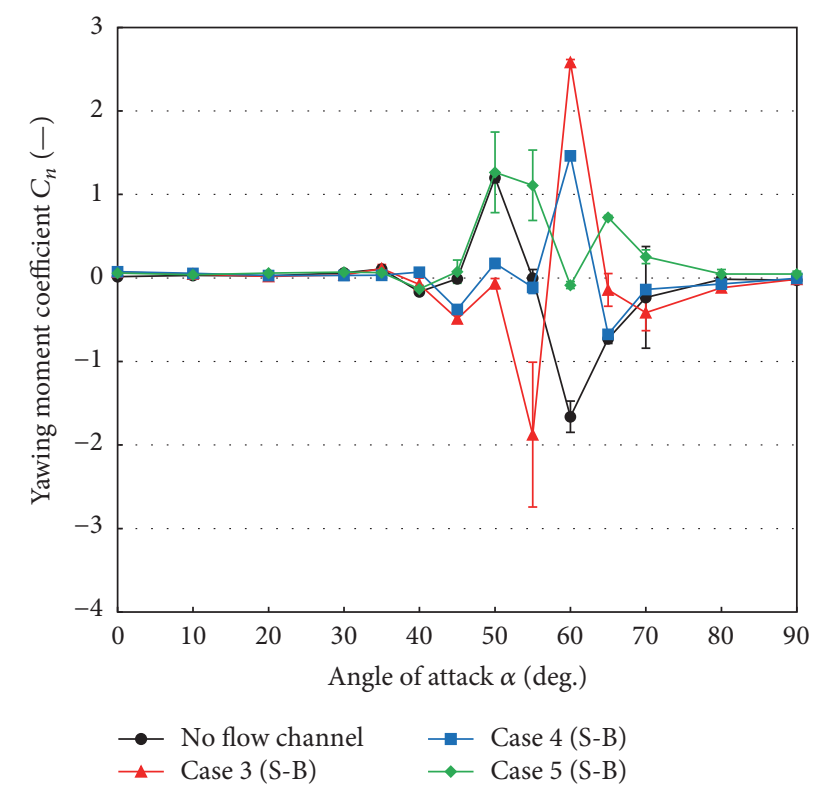

FIgURE 20: Yawing moment coefficient: S-B type channels, $16 \mathrm{~m} / \mathrm{s}$.

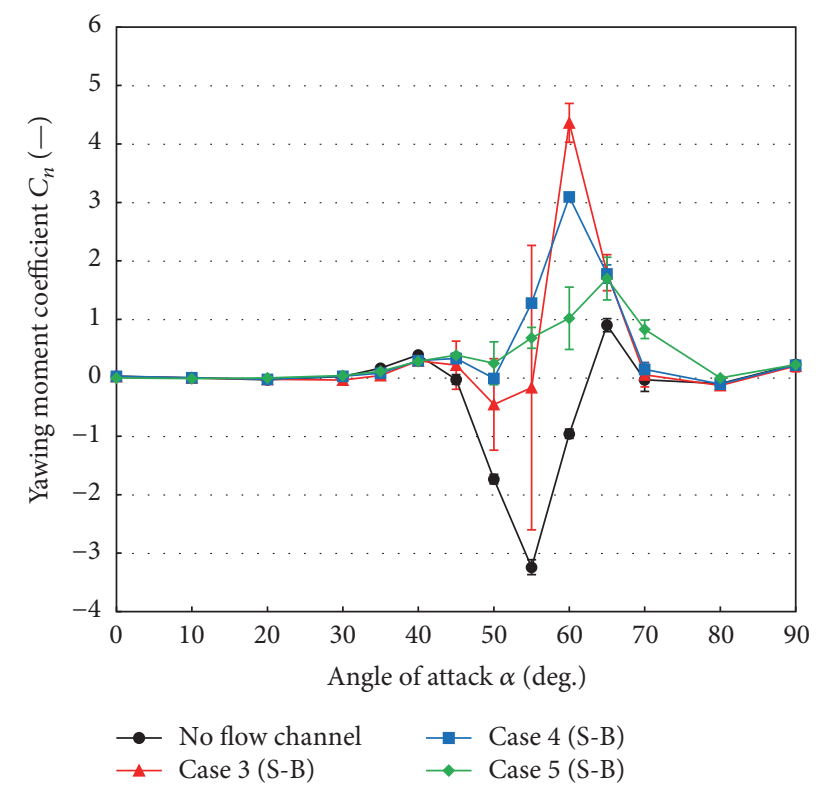

FIGURE 21: Yawing moment coefficient: S-B type channels, $35 \mathrm{~m} / \mathrm{s}$.

\section{Conclusions}

In this study, we proposed installing penetrating flow channels to the apex of a slender body aiming to suppress the side force and yawing moment at high angle of attack. In order to verify our concept, we experimentally investigated the aerodynamic characteristics and visualized the surface flow pattern by the oil flow method in the low-speed wind tunnel, and following conclusions were obtained.

(1) It was successfully confirmed that both the B-B (Blowing-Blowing) type and S-B (Suction-Blowing) type flow channel work to suppress the side force and yawing moment.

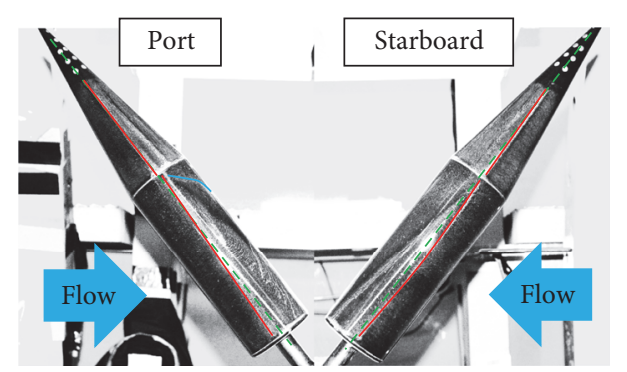

Figure 22: Surface flow patterns: port and starboard side view, without flow channel.

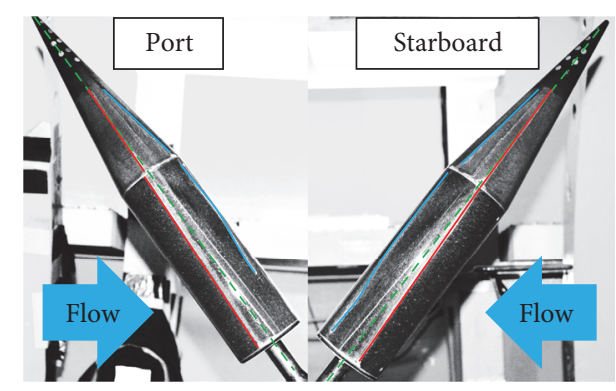

FIGURE 23: Surface flow patterns: port and starboard side view, with the S-B type channel.

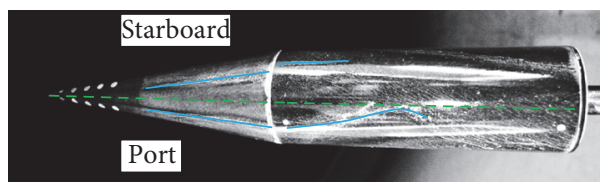

FIGURE 24: Surface flow patterns: back side view, without flow channel.

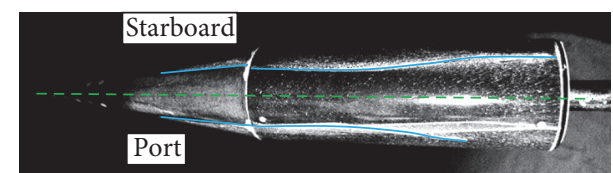

FIGURE 25: Surface flow patterns: back side view, with the S-B type channel.

(2) For the B-B type flow channel, the suppression effects depend on the mainstream flow velocity, and therefore, installation position and channel shape ( $\mathrm{T}$ or $\mathrm{Y}$ shape) need to be selected in response to the mainstream condition. In the mainstream of $16 \mathrm{~m} / \mathrm{s}$, the $\mathrm{Y}$ shape channel at $3.5 \%$ length from the model tip is the most effective, and the suppression rate is up to about $59 \%$ and $49 \%$ for the side force and yawing moment, respectively. In the mainstream of $24 \mathrm{~m} / \mathrm{s}$, the T-shape channel at $10 \%$ length is the most effective, and the suppression rate is up to $19 \%$ and $29 \%$ for the side force and yawing moment, respectively.

(3) For the S-B type flow channel, the channel at 17\% length from the model tip is the most effective in the mainstream of 16 and $35 \mathrm{~m} / \mathrm{s}$. The suppression rate of 
the side force is up to 45 and $64 \%$ in 16 and $35 \mathrm{~m} / \mathrm{s}$, respectively. The suppression rate of the yawing moment is up to 24 and $48 \%$ in 16 and $35 \mathrm{~m} / \mathrm{s}$, respectively.

(4) The B-B type flow channel reduces its ability in higher mainstream flow velocity. On the other hand, the S-B type can keep its ability at high level even in higher mainstream flow velocity, and in addition, the effective installation position is not strongly affected by the mainstream flow velocity. Therefore, it can be concluded that the S-B type is more attractive for suppression of the high-angle-of-attack side force and yawing moment.

\section{Nomenclature}

$\alpha$ : Angle of attack

$C_{N}$ : Normal force coefficient

$C_{Y}$ : Side force coefficient

$C_{n}$ : Yawing moment coefficient.

\section{Competing Interests}

The authors declare that they have no competing interests.

\section{Acknowledgments}

This research was partially supported by the Grant-in-Aid for Young Scientists (A) K116H06133 of the Japan Society for the Promotion of Science.

\section{References}

[1] B. L. Hunt, "Asymmetric vortex forces and wakes on slender bodies," in Proceedings of the 9th AIAA Atmospheric Flight Mechanics Conference, AIAA paper 82-1336, San Diego, Calif, USA, 1982.

[2] D. H. Bridges, “The asymmetric vortex wake problem-asking the right question," in Proceedings of the 36th AIAA Fluid Dynamics Confernce and Exhibit, AIAA Paper 2006-3553, pp. 1737-1765, San Francisco, Calif, USA, June 2006.

[3] D. R. Williams, "A review of forebody vortex control scenarios," in Proceedings of the 28th AIAA Fluid Dynamics Conference, AIAA Paper 97-1967, June-July 1997.

[4] G. N. Malcolm, "Forebody vortex control-a progress review," AIAA Paper 93-3540, 1993.

[5] J. E. Bernhardt and D. R. Williams, "Proportional control of asymmetric forebody vortices," AIAA Journal, vol. 36, no. 11, pp. 2087-2093, 1998.

[6] F. W. Roos, "Microblowing: an effective, efficient method of vortex-asymmetry management," in Proceedings of the 18th AIAA Applied Aerodynamics Conference, AIAA paper 20004416, Denver, Colo, USA, August 2000.

[7] F. W. Roos, "Microblowing for high-angle-of-attack vortex flow control on a fighter aircraft," Journal of Aircraft, vol. 38, no. 3, pp. 454-457, 2001.

[8] F. O. Thomas, T. C. Corke, M. Iqbal, A. Kozlov, and D. Schatzman, "Optimization of dielectric barrier discharge plasma actuators for active aerodynamic flow control," AIAA Journal, vol. 47, no. 9, pp. 2169-2178, 2009.
[9] E. Moreau, R. Sosa, and G. Artana, "Electric wind produced by surface plasma actuators: a new dielectric barrier discharge based on a three-electrode geometry," Journal of Physics D: Applied Physics, vol. 41, no. 11, Article ID 115204, 2008.

[10] F. Liu, S. Luo, C. Gao et al., "Flow control over a conical forebody using duty-cycled plasma actuators," AIAA Journal, vol. 46, no. 11, pp. 2969-2973, 2008.

[11] H. Hu, H. Li, X. Meng, X. Yan, F. Liu, and S. Luo, "Optimization of dielectric barrier discharge plasma actuators for conicalforebody flow control," in Proceedings of the 47th AIAA Plasmadynamics and Lasers Conference, AIAA 2016-4018, Washington, DC, USA, 2016.

[12] H. Nishida, S. Mizuki, I. Miyazaki, S. Nonaka, T. Nonomura, and Y. Inatani, "Preliminary experimental study on aerodynamic characteristics control of slender body using DBD plasma actuator," Transaction of JSASS Aerospace Technology Japan, vol. 10, pp. Pe_97-Pe_103, 2012.

[13] M. Satoh, H. Nishida, and T. Nonomura, "Numerical study of vortex flow control on high-angle-of-attack slender body," Transaction of JSASS Aerospace Technology Japan, vol. 12, no. ists29, pp. Pe43-Pe49, 2014.

[14] T. Igarashi, "Flow characteristics around a circular cylinder with a slit: I-flow control and flow patterns," Bulletin of the JSME, vol. 21, pp. 656-664, 1978.

[15] T. Igarashi, "Flow characteristics around a circular cylinder with a slit: 2nd report, effect of boundary layer suction," Bulletin of the JSME, vol. 25, no. 207, pp. 1389-1397, 1982.

[16] H. J. Allen and E. W. Perkins, "Characteristics of flow over slender inclined bodies at revolution," NACA RM A 50 L 07, 1951.

[17] E. R. Keener, "Oil flow separation patterns on an ogive forebody," AIAA Journal, vol. 21, no. 4, pp. 550-556, 1983.

[18] D. Degani and Y. Levy, "Asymmetric turbulent vortical flows over slender bodies," AIAA Journal, vol. 30, no. 9, pp. 2267-2273, 1992. 


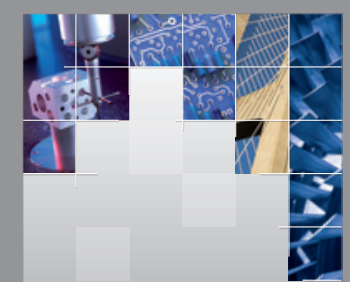

\section{Enfincering}
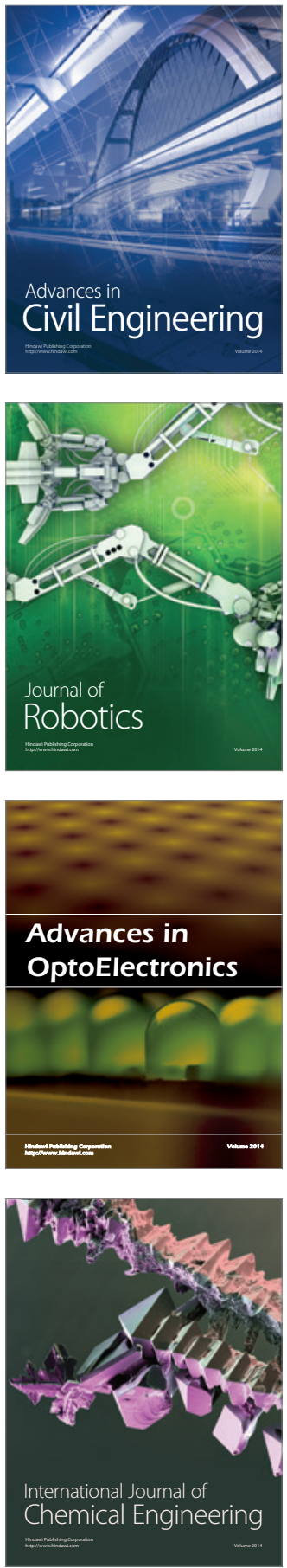

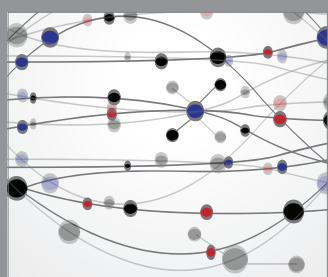

The Scientific World Journal

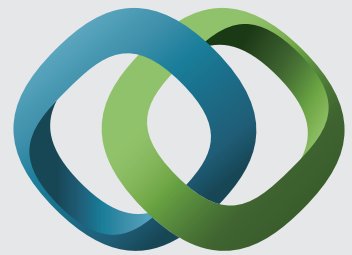

\section{Hindawi}

Submit your manuscripts at

http://www.hindawi.com
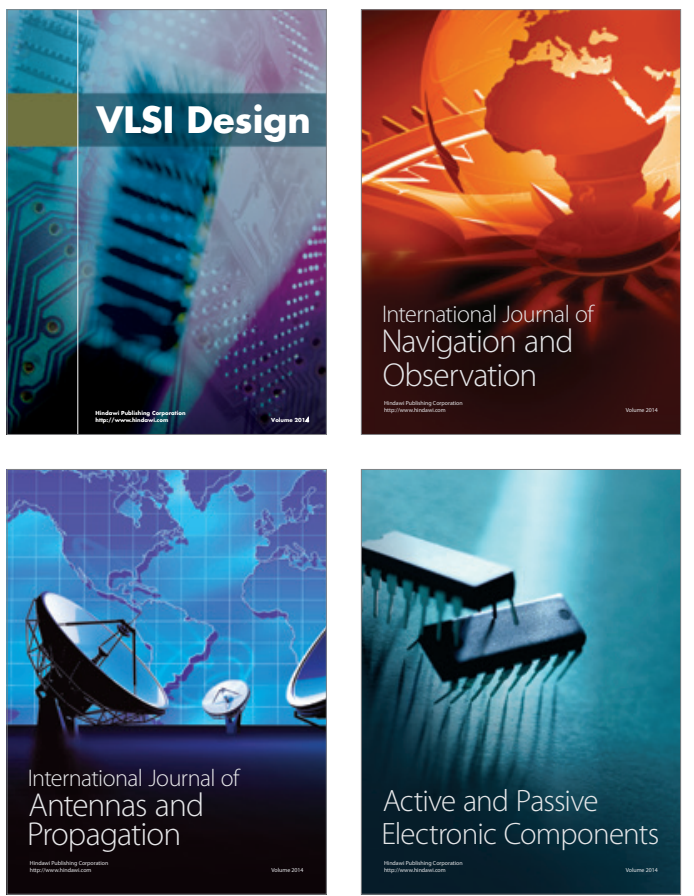
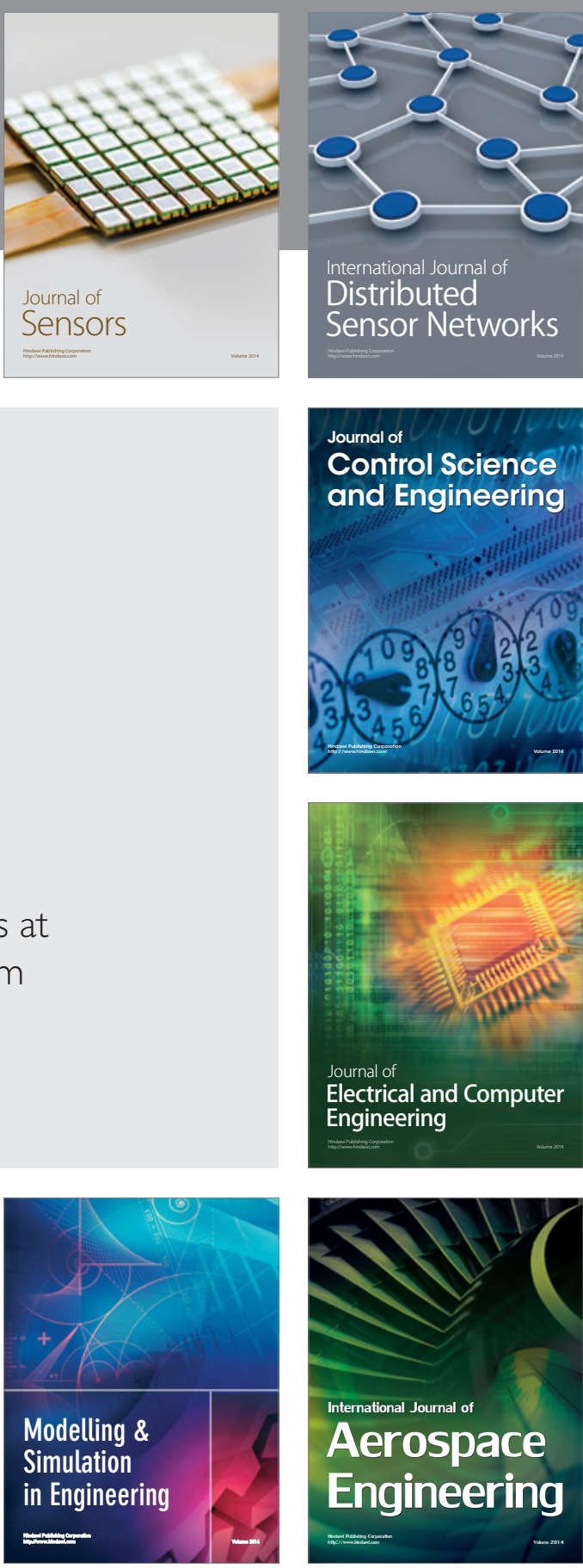

International Journal of

Distributed

Sensor Networks

Journal of

Control Science

and Engineering
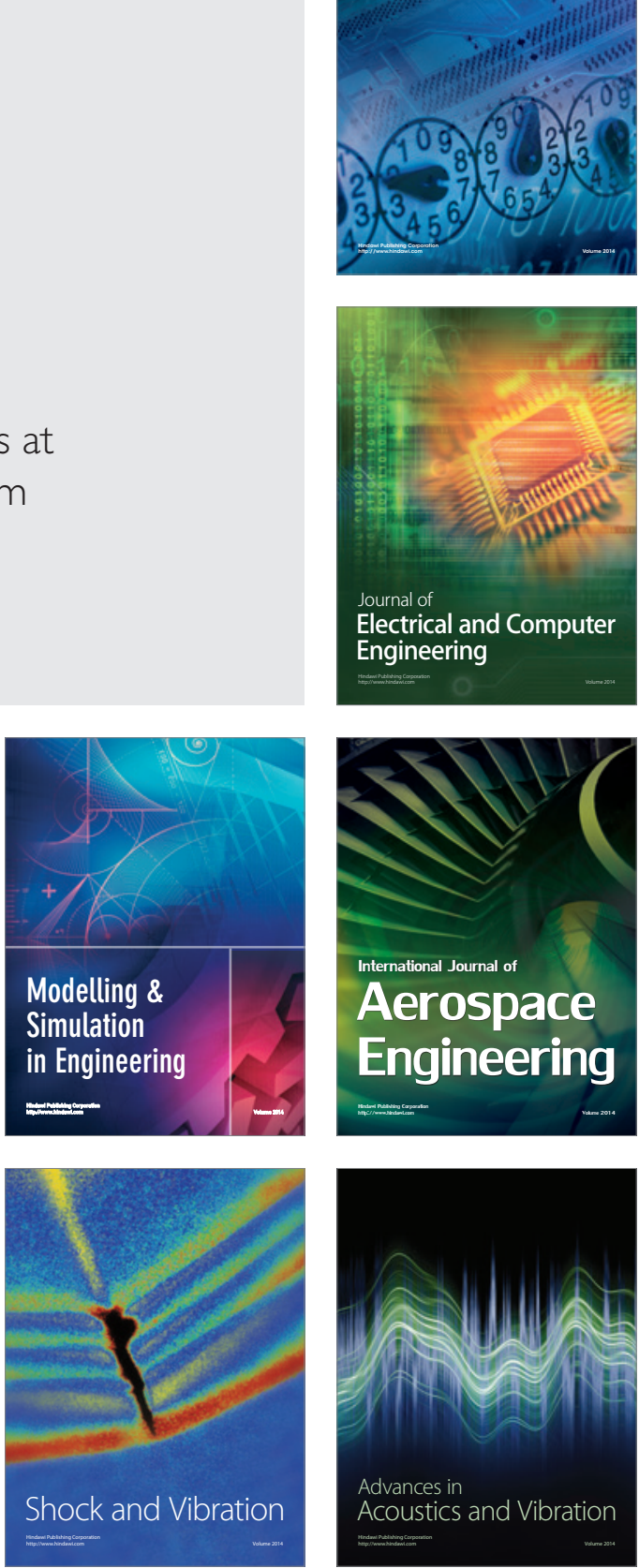